\title{
An inexpensive universal feeder suitable for small numbers of large rations
}

\author{
C. DONALD HETH \\ University of Alberta, Edmonton, Alberta T6G 2E9, Canada
}

\begin{abstract}
This report describes a simple universal feeder designed for operant conditioning applications in zoo settings. The apparatus delivers up to six large rations of food. It is portable, easily serviced, and can be constructed in a modest carpentry shop.
\end{abstract}

There has been considerable interest in the application of operant conditioning procedures in zoological parks and preserves (e.g., Markowitz, 1975). Many of these applications involve the maintenance of instrumental contingencies within an animal's enclosure, making the use of automated equipment desirable.

In developing a program of this nature at the Edmonton Valley Zoo, we encountered a number of constraints concerning the use of an automated feeder. To avoid disruption of an animal's normal feeding regimen, we wanted to deliver a small number of relatively large rations of the animal's normal diet (e.g., hamburger patties). A battery-powered device was required, since many of the animal sites lacked ac power installations. Finally, we wanted a system that could be easily serviced by zoo personnel. Since most commercially available universal feeders are designed for use in the laboratory, they did not meet our particular conjunction of requirements. The feeder described here was developed to meet these specifica. tions. Its simplicity and portability may prove useful in similar field applications of operant conditioning.

Feeder. The feeder is hexagonal in shape, with walls radiating in from the vertices forming six compartments. The floor of each compartment is a trap door hinged on the perimeter wall. The doors are latched by magnets (Edmund P-40637; Edmund Scientific Company, Edscorp Building, Barrington, New Iersey 08007) affixed to each door and wall at the central hub. This arrangement is depicted in Figure 1.

The latches are sprung by a small wedge glued to the underside of a rotating disk on the bottom of the feeder. Each door is fitted with a small flange that projects across the circular path of the wedge. As the wedge travels over the flange, the flange is pushed downward, breaking the contact between the two magnets. This sequence is depicted in the right-hand side of the bottom panel of Figure 1. The weight of the ration in the compartment then forces the door

The development of this device was supported by a grant from the Alma Mater Fund of the University of Alberta. I thank R. Wood, P. DeGroot, J. Kisilevich, and E. Heth for their help. open. In this way, the six compartments can be opened sequentially. After opening, the doors hang vertically. Hence, the feeder can accommodate any
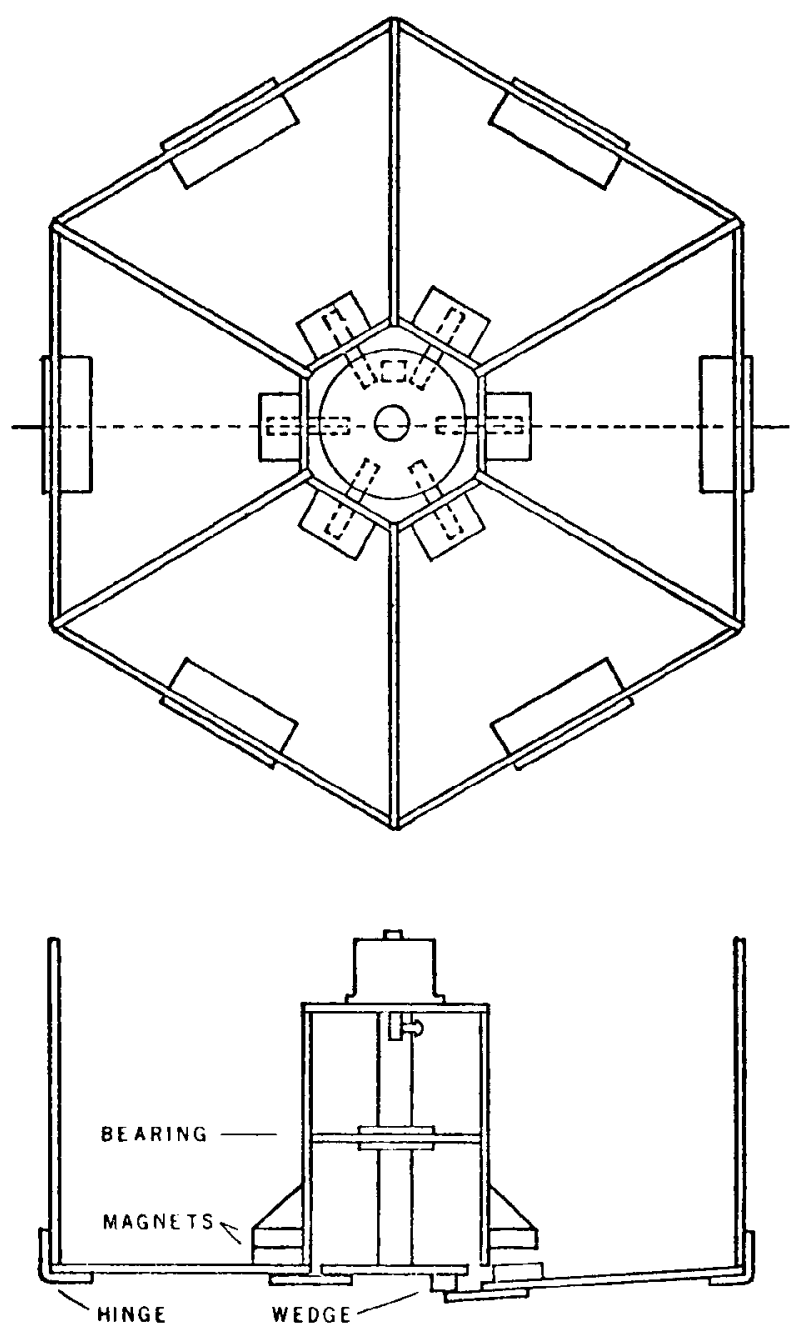

Figure 1. Top panel: Top view of the feeder. The releasing wedge is shown in the resting position between the top two compartments. Bottom panel: Gross-sectional view of the feeder along the dotted line of the top panel. The releasing wedge has been rotated to the releasing position for the door on the right. 
substance that will not adhere to a vertical surface.

The disk is rotated by a shaft mounted inside the hub of the hexagon. This shaft is driven by a $24-\mathrm{V}$ dc stepping motor (Ledex Digimotor 213229-028; Ledex Inc., 123 Webster Street, Dayton, Ohio 45402) with 30-deg steps. Thus, each compartment is unlatched with two steps of the motor: The first steps the wedge to the flange; the second steps it past the flange and to the resting position before the next flange. The motor should be aligned such that the wedge is initially between two flanges. By using a set screw on the motor shaft and a bolt-on mount, the motor can be removed and the entire feeder placed in a commercial dishwasher for cleaning.

The motor can be driven with lantern batteries or rechargeable wet cells (two motorcycle batteries are reasonably portable). The feeder requires two pulses for each cycle, and these must be programmed externally.

To load the feeder, the doors are merely snapped in place. The disk is rotated such that the wedge is in the resting position before the first flange. Each compartment can then be loaded from the top.

The prototype depicted in Figure 1 was constructed of $.3-\mathrm{cm}$ thickness sheets of acrylic plastic. The trapazoidal doors have sides of $11.4 \mathrm{~cm}$ and a top and bottom of $3.8 \mathrm{~cm}$ and $15.2 \mathrm{~cm}$, respectively. These dimensions are designed for food rations of about $200 \mathrm{~g}$. Rations within this range are light enough not to spontaneously trip the latch and heavy enough to continue the unlatching motion once magnetic contact is broken. A lighter load can be accommodated by weighting the doors. A greater capacity can be built in by using more powerful magnets on the latches. The magnets of our prototype were cemented to the walls and doors. Since it is sometimes difficult to bind metal to acrylic plastic, the magnets can be attached by bolts. The shaft is a $1.3-\mathrm{cm}$ plastic dowel that runs through a hole drilled in a plastic strut glued across the hub of the hexagon. Two rings cut from $1.3-\mathrm{cm}$ inside diameter plastic tubing and glued to the shaft on either side of this strut form the bearing. The wedge is a rounded piece of plastic, $.4 \mathrm{~cm}$ high, cut from a dowel. The wedge and the door flanges are beveled slightly to facilitate the unlatching motion.

\section{REFERENCE}

Markowtrz, $H$. Analysis and control of behavior in the zoo. In National Academy of Sciences (Ed.), Research in zoos and aquariums: A symposium. Washington, D.C: Author, 1975.

(Received for publication July 18, 1978; revision accepted September 28, 1978.) 